\title{
REGULAR REES MATRIX SEMIGROUPS AND REGULAR DUBREIL-JACOTIN SEMIGROUPS
}

\author{
D. B. MCALISTER
}

(Received 30 July 1980)

Communicated by T. E. Hall

\begin{abstract}
A partially ordered semigroup $S$ is said to be a Dubreil-Jacotin semigroup if there is an isotone homomorphism $\theta$ of $S$ onto a partially ordered group such that $\left\{x \in S: x^{2} \theta<x \theta\right\}$ has a greatest member. In this paper we investigate the structure of regular Dubreil-Jacotin semigroups in which the imposed partial order extends the natural partial order on the idempotents. The main tool used is a local structure theorem which is introduced in Section 2. This local structure theorem applies to many other contexts as well.
\end{abstract}

1980 Mathematics subject classification (Amer. Math. Soc.): 20 M 10.

\section{Partially ordered regular semigroups}

It is well known that the idempotents of a regular semigroup $S$ can be partially ordered by setting

$$
e \omega f \text { if and only if } e=e f=f e .
$$

This partial order is called the natural partial order on the idempotents of $S$. We shall say that a partially ordered semigroup $S$ is naturally partially ordered if the imposed partial order $<$ extends the natural partial order on the idempotents; that is, if

$$
e=e f=f e \quad \text { implies } e<f .
$$

Note there is no assumption in the definition that $e \leqslant f$ implies $e=e f=f e$. As we shall see, the existence of a partial order, extending the natural partial

This research was supported by a grant from the National Science Foundation. 
order on the idempotents, imposes significant structural limitations on regular semigroups.

K. S. S. Nambooripad [10] has shown that the relation < defined by:

$$
a \leqslant b \text { if and only if } a=a a^{\prime} b \text { and } a \in b S,
$$

for some inverse $a^{\prime}$ of $a$ in $S$, is a partial order on any regular semigroup $S$. This partial order extends the natural partial order on the idempotents of $S$ but, in general, it is not compatible with multiplication. Indeed, Nambooripad shows that $<$ turns $S$ into a partially ordered semigroup if and only if $S$ obeys one, and thus all, of the conditions described in Lemma 1.1.

LEMMA 1.1. Let $S$ be a regular semigroup. Then the following conditions on $S$ are equivalent:

i) $|S(e, f)|=1$ for each $e^{2}=e, f^{2}=f \in S$;

ii) $e S e$ is inverse for each idempotent $e \in S$;

iii) $S$ does not contain a copy of $B^{1}$ for any non trivial rectangular band $B$.

The notation $S(e, f)$ in Lemma 1.1 denotes the sandwich set of $e$ and $f$; thus $S(e, f)=\left\{g^{2}=g \in S: e g f=e f\right.$ and $\left.g e=g=f g\right\}$. The importance of the sandwich set stems from the following useful observation, due to Nambooripad [9]. In this lemma, $V(a)$ denotes the set of inverses of $a$.

LEMMA 1.2. Let $S$ be a regular semigroup and let $a, b \in S$; let $a^{\prime} \in V(a)$, $b^{\prime} \in V(b)$. Then, for each $g \in S\left(a^{\prime} a, b b^{\prime}\right), b^{\prime} g a^{\prime} \in V(a b)$.

COROLlaRY 1.3. Let $S$ be a regular semigroup and let $x_{i} \in S, 1<i<n$; let $x_{1}^{\prime} \in V\left(x_{1}\right), x_{n}^{\prime} \in V\left(x_{n}\right)$. Then $V\left(x_{1} x_{2} \cdots x_{n}\right) \cap x_{n}^{\prime} S x_{1}^{\prime} \neq \varnothing$.

Because of condition (ii), in Lemma 1.1, we shall say that a regular semigroup is locally inverse if it obeys any one of the conditions of Lemma 1.1. The subsemigroups $e S e, e^{2}=e$, are called the local submonoids of $S$. Nambooripad [10] used the term pseudo inverse to describe locally inverse semigroups.

Proposition 1.4. Let $S$ be a regular semigroup. Then $S$ can be turned into a partially ordered semigroup, in which the imposed partial order extends the natural partial order on the idempotents, if and only if $S$ is locally inverse.

Proof. Nambooripad's result [10], quoted above, shows that the relation $a \leqslant b$ if and only if $a=a a^{\prime} b$ and $a \in b S$, for some $a^{\prime} \in V(a)$, turns $S$ into a naturally partially ordered semigroup provided $S$ is locally inverse. 
Conversely, suppose that $S$ is a naturally partially ordered semigroup under $<$ and let $u, v \in e S e$ where $u, v, e$ are idempotents and, say, $u \Re v$. Then $u$, vwe imply $u, v<e$. But then $u \Re v$ implies $u=v u<v e=v$ and $v=u v<u e=u$ so that $u=v$. Hence each $\Re$-class of $e S e$ contains exactly one idempotent. Similarly, each $\mathcal{L}$-class contains exactly one idempotent. Hence $e S e$ is inverse.

Proposition 1.5. Let $S$ be a partially ordered regular semigroup.

i) If $S$ is naturally ordered then $f \leqslant e$ implies $f=f e f$.

ii) If $S$ has a greatest idempotent $u$ then $S$ is naturally ordered if and only if $f=$ fuf for each idempotent $f \in S$.

Proof. Suppose that $u, v$ are idempotents of a partially ordered semigroup $S$ and let $u<v$. Then

$$
u v \cdot u v=u v \cdot u \cdot v \leqslant u v \cdot v \cdot v=u v=u \cdot u \cdot u v \leqslant u \cdot v \cdot u v=u v \cdot u v
$$

so that $u v$ is idempotent; similarly $v u$ is idempotent. Further $u<v u$ so that $u \cdot v u=u v u$ is idempotent.

i) Suppose now that $<$ extends $\omega$ and that $f<e$. Then $f e f$ is idempotent and $f<f e f$. But $f e f$ idempotent implies $f e f \omega f$ and so, because $<$ extends $\omega, f e f<f$. Hence $f=f e f$.

ii) Suppose that $S$ has a greatest idempotent $u$; then $f<u$ for each idempotent $f$. Hence $f=f u f$ provided that $S$ is naturally ordered. Conversely, suppose $v=v u v$ for each idempotent $v$ and let $e, f$ be idempotents with $e \omega f$. Then $e \leqslant u$ implies eue $<u$ so

$$
e=e u e=\text { feuef }<\text { fuf }=f .
$$

Thus < extends $\omega$ and $S$ is naturally ordered.

COROLLARY 1.6. Let $S$ be a naturally ordered regular semigroup with a greatest idempotent $u$. Then $S=S u S$.

Proof. Let $a \in S, a^{\prime} \in V(a)$. Then $a a^{\prime}=a a^{\prime} u a a^{\prime}$ so that $a=a a^{\prime} u a \in S u S$.

Corollary 1.6 is relevant to our present investigations because any regular Dubreil-Jacotin semigroup $S$ has a greatest idempotent. Indeed, suppose that $\theta$ is an isotone homomorphism onto a partially ordered group $G$ and let $u=$ $\max \left\{x \in S: x^{2} \theta<x \theta\right\}$. Then, for $u^{\prime} \in V(u)$ we have $u u^{\prime}<u$, since $u u^{\prime}$ is idempotent, so that $1=\left(u u^{\prime}\right) \theta<u \theta \leqslant 1$, where 1 denotes the identity of $G$. Hence $u \in 1 \theta^{-1}$ which is, consequently, a partially ordered regular semigroup with a greatest element $u$. Lemma 1.7 shows that $u$ is an idempotent of $S$. 
LEMMA 1.7 [8]. Let $S$ be a partially ordered regular semigroup with a greatest element $u$. Then $u^{2}=u$ and $u S u$ is a semilattice. Further the imposed partial order on $S$ coincides with the natural partial order.

In a sequence of papers [2], [3], [4], [5] T. S. Blyth has investigated the structure of various classes of regular Dubreil-Jacotin semigroups. These are perfect in the sense of [5] and Blyth shows, [5] equations (2) and (10), that $e=e u e$ for each idempotent $e$, where $u$ denotes the greatest idempotent. Hence we have

COROLlary 1.8. Let $S$ be a perfect Dubreil-Jacotin semigroup. Then the imposed partial order extends the natural partial order on the idempotents of $S$. Further, if $u$ denotes the greatest idempotent of $S$ then $S=S u S$.

Naturally ordered regular semigroups having a greatest idempotent have many properties analogous to those of the orthodox Dubreil-Jacotin semigroups considered in [3], [4], [8]. For example, each element in such a semigroup $S$ has a greatest inverse. [Let $u$ denote the greatest idempotent of $S$ and let $x \in S$, $x^{\prime} \in V(x)$. Then, since $e=e u e$ for each idempotent $e, u x^{\prime} u \in V(x)$. Further, for any $x^{\prime \prime} \in V(x)$ we have $x^{\prime \prime}=x^{\prime \prime} x x^{\prime} x x^{\prime \prime} \leqslant u x^{\prime} u$.] However, [8], Theorem 5.5 shows that these semi-groups need not be orthodox. Indeed, we have the following proposition which generalizes [5], Theorem 2 .

PROPOSITION 1.9. Let $S$ be a partially ordered regular semigroup with a greatest idempotent $u$. Then the following are equivalent:

(a) $S$ is naturally ordered and orthodox;

(b) $u$ is a middle unit, that is $x u y=x y$ for each $x, y \in S$.

Proof. Suppose that (a) holds. Then, by Proposition 1.5, $e=e$ eue for each idempotent $e \in S$. Hence, by [8], Theorem 5.4, $u$ is a middle unit of $S$.

Suppose, on the other hand, that (b) holds and let $B$ be the subsemigroup generated by the idempotents of $S$. Then $u$ is the greatest element of $B$ and $x^{2}=x u x$ for each $x \in B$. Hence, by [8], Corollary 5.6, $B$ is a band. Thus $B$ is orthodox and, by Proposition 1.5, it is naturally ordered.

\section{The local structure theorem}

Let $S$ be a regular semigroup and let $I, \Lambda$ be sets; let $P$ be a $\Lambda \times I$ matrix over $S$. Then the set of all triples $(i, s, \lambda) \in I \times S \times \Lambda$ is a semigroup under the multiplication

$$
(i, s, \lambda)(j, t, \mu)=\left(i, s p_{\lambda j} t, \mu\right)
$$


This semigroup, which is denoted by $\mathscr{N}(S ; I, \Lambda ; P)$, is not, in general, regular. However, as we shall see, the set of regular elements forms a subsemigroup; thus a regular semigroup. We shall denote it by $\Re \Re(S ; I, \Lambda P)$ and call it a regular Rees matrix semigroup over $S$. If $S=S^{1}$ and 1 is an entry of $P$, then we say that $\Re \Re(S ; I, \Lambda ; P)$ is unital.

LeMMA 2.1. Let $S$ be a regular semigroup, $I, \Lambda$ sets and let $P$ be a $\Lambda \times I$ matrix over $S$. Then

(i) $(i, s, \lambda) \in \mathscr{R}(S ; I, \Lambda ; P)$ is regular if and only if $V(s) \cap p_{\lambda j} S p_{\mu i} \neq \varnothing$ for some $j \in I, \mu \in \Lambda$;

(ii) $\Re \mathscr{R}(S ; I, \Lambda ; P)=\left\{(i, s, \lambda): V(s) \cap p_{\lambda j} S p_{\mu j} \neq \varnothing\right.$ for some $\left.j \in I, \mu \in \Lambda\right\}$ is a regular subsemigroup of $\mathfrak{T}(S ; I, \Lambda ; P)$.

Proof. Let $(j, t, \mu) \in \mathscr{R}(S ; I, \Lambda ; P)$; then $(j, t, \mu) \in V((i, s, \lambda))$ implies $p_{\lambda j} t p_{\mu i} \in V(s)$. Conversely, if $p_{\lambda j} t p_{\mu i} \in V(s)$ then $(i, s, \lambda)(j, t, \mu)(i, s, \lambda)=$ $(i, s, \lambda)$. Hence (i) holds.

(ii) Suppose $(i, s, \lambda),(j, t, \mu)$ are regular. Then, by (i), there exist $s^{\prime} \in V(s) \cap$ $p_{\lambda k} S p_{v i}, t^{\prime} \in V(t) \cap p_{\mu h} S p_{\rho j}$ for some $k, h \in I, \mu, \rho \in \Lambda$. Hence, by Corollary $1.3, V\left(s p_{\lambda j} t\right) \cap t^{\prime} S s^{\prime} \neq \varnothing$; thus $V\left(s p_{\lambda j} t\right) \cap p_{\mu h} S p_{\nu i} \neq \varnothing$. Since $(i, s, \lambda)(j, t, \mu)=$ $\left(i, s p_{\lambda}:, \mu\right)$ this implies $(i, s, \lambda)(j, t, \mu)$ is regular.

ExAmple 2.2. Let $S$ be the chain $\{1>a>b>0\}, I=\Lambda\{1,2\}$ and let $P$ be the $2 \times 2$ matrix $\left(\begin{array}{ll}1 & a \\ b & 0\end{array}\right)$. Then $(i, s, \lambda) \in \Re \mathscr{R}(S ; I, \Lambda ; P)$ if and only if $s=s^{\prime} \in$ $p_{\lambda j} S p_{\mu i}$ for some $j \in I, \mu \in \Lambda$. Since multiplication in $S$ is $\mathrm{min}$, it follows that $(i, s, \lambda) \in \mathscr{R} \mathfrak{N}(S ; I, \Lambda ; P)$ if and only if $s<p_{\lambda j} p_{\mu j}$. That is, $s$ is less than the $\lambda$, $i$ entry of $P^{2}$ regarded as a matrix over the lattice $S$. Since $P^{2}=\left(\begin{array}{cc}1 & a \\ b & b\end{array}\right)$ we find that $\Re \mathscr{N}(S ; I, \Lambda ; P)$ has eleven elements, whereas $\mathscr{R}(S ; I, \Lambda ; P)$ has 16 ;

$$
\begin{array}{r}
(1,1,1) \\
(1, a, 1),(2, a, 1) \\
(1, b, 1),(1, b, 2),(2, b, 1),(2, b, 2)^{*} \\
(1,0,1),(1,0,2),(2,0,1),(2,0,2) .
\end{array}
$$

Only $(2, b, 2)$ is not idempotent.

We say that a homomorphism $\theta$ of a regular semigroup $S$ onto a regular semigroup $T$ is a local isomorphism if $\theta$ maps each $e S e, e^{2}=e$, isomorphically into $T$; in this case, we call $T$ a locally isomorphic image of $S$. 
LEMMA 2.3. Let $\theta$ be a homomorphism of a regular semigroup $S$ onto a regular semigroup $T$.

(i) If $\theta$ is a local isomorphism then each idempotent $\theta \circ \theta^{-1}$ class is a rectangular band.

(ii) If $S$ is locally inverse, and each idempotent $\theta \circ \theta^{-1}$ class is a rectangular band, then $\theta$ is a local isomorphism.

Proof. (i) Nambooripad [10] shows that every idempotent class of a congruence $\rho$ on $S$, is completely simple if and only if $e \omega f$, epf together imply $e=f$. Suppose $e \theta=f \theta$ where $e, f$ are idempotents and $e \omega f$ then, since $\theta$ is one to one on $f S f, e=f$. Hence each idempotent $\theta \circ \theta^{-1}$ class is completely simple. Further, since $\theta$ is one to one on each $f S f$, these classes have trivial subgroups. That is, they are rectangular bands.

(ii) Let $a, b \in e S e$, where $e^{2}=e$, and suppose that $a \theta=b \theta$. Then, for each $a^{\prime} \in V(a) \cap e S e$, we have $a a^{\prime} \theta=b a^{\prime} \theta$. Thus $a a^{\prime}$ and $b a^{\prime}$ belong to a rectangular sub-band of $e S e$. Since $e S e$ is inverse, this implies $a a^{\prime}=b a^{\prime}$ so that $a=b a^{\prime} a \in$

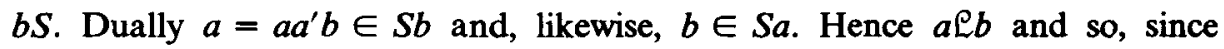
$a=b a^{\prime} a, a=b$.

TheOREM 2.4. (Local Structure Theorem.) Let $S$ be a regular semigroup and suppose that $S=S e S$ for some idempotent $e$. Then $S$ is a locally isomorphic image of a unital regular Rees matrix semigroup over $e S e$.

Proof. For each idempotent $u \in S$, there exists $r_{u} \in S, r_{u}^{\prime} \in V\left(r_{u}\right)$ such that $r_{u} r_{u}^{\prime}=u$ and $r_{u}^{\prime} r_{u} \omega e$, since $u \in S e S$. For each pair of idempotents $v$, $u$, let $p_{v, u}=r_{v}^{\prime} r_{u}$; then, since $r_{v}^{\prime} r_{v}, r_{u}^{\prime} r_{u} \omega e$, we have $p_{v, u} \in e S e$. We may further assume, without loss of generality, that $r_{e}^{\prime}=r_{e}=e$.

Let $I$ be a set of idempotents of $S$, such that for each idempotent $v \in S$ there exists $u \in I$ with $v=u v$. Likewise, let $\Lambda$ be a set of idempotents such that for each $v^{2}=v \in S$ there exists $u \in \Lambda$ with $v=v u$ and let $P$ be the $\Lambda \times I$ matrix with entries $p_{v, u}$. Then we can form the (unital) regular Rees matrix semigroup $\Re \mathfrak{R}(e S e ; I, \Lambda ; P)$ over $e S e$. Its entries consist of all triples $(u, s, v)$ in $I \times e S e$ $\times \Lambda$ for which $V(s) \cap r_{v}^{\prime} r_{v} S r_{u}^{\prime} r_{u} \neq \varnothing$, since $r_{v}^{\prime} r_{v} e=r_{v}^{\prime} r_{v}, e r_{u}^{\prime} r_{u}=r_{u}^{\prime} r_{u}$.

Define $\theta: \Re \mathscr{R}(e S e ; I, \Lambda ; P) \rightarrow S$ by $(u, s, v) \theta=r_{u} s r_{v}^{\prime}$. Then

$$
\begin{aligned}
((u, s, v)(x, t, y)) \theta & =\left(u, s p_{v, x} t, y\right) \theta=r_{u} s p_{v, x} t r_{y}^{\prime} \\
& =r_{u} s r_{v}^{\prime} r_{x} t r_{y}^{\prime}=(u, s, v) \theta(x, t, y) \theta
\end{aligned}
$$

so that $\theta$ is a homomorphism of $T=\mathscr{R} \mathfrak{R}(e S e ; I, \Lambda ; P)$ into $S$.

Now, let $s \in S$. Then, because of the choice of $I, \Lambda, s \in u S v$ for some $u \in I$, $v \in \Lambda$ so that $s=r_{u} r_{u}^{\prime} s r_{v} r_{v}^{\prime}=r_{u} t r_{v}^{\prime}$ where $t=r_{u}^{\prime} s r_{v}$. By Corollary $1.3, t \in e S e$ 
has an inverse in $r_{v}^{\prime} S r_{u}=r_{v}^{\prime} r_{v} S r_{u}^{\prime} r_{u}=r_{v}^{\prime} r_{w} S r_{z}^{\prime} r_{u}$ for some $w \in I, z \in \Lambda$. Hence $(u, t, v) \in \Re \mathfrak{R}(e S e ; I, \Lambda ; P)$. But $(u, t, v) \theta=r_{u} t r_{v}=s$. Hence $\theta$ is onto.

Finally, suppose that $(u, s, v)$ is idempotent. Then $(x, t, y) \in$ $(u, s, v) T(u, s, v)$ implies $x=u, y=v$ and $t=t p_{v, u} s=s p_{v, u} t$; further $(x, t, y) \theta$ $=r_{u} t r_{v}^{\prime}$. Suppose $(u, t, v),(u, z, v) \in(u, s, v) T(u, s, v)$ and $(u, t, v) \theta=$ $(u, z, v) \theta$; then $r_{u} t r_{v}^{\prime}=r_{u} z r_{v}^{\prime}$ which, in turn, implies

$$
\begin{aligned}
t & =s p_{v, u} t p_{v, u} s=s r_{v}^{\prime} r_{u} t r_{v}^{\prime} r_{u} s \\
& =s r_{v}^{\prime} r_{u} z r_{r}^{\prime} r_{u} s=z .
\end{aligned}
$$

Thus $\theta$ is one to one on each local submonoid. That is, $\theta$ is a local isomorphism.

COROllary 2.5. Let $S$ be a regular semigroup and suppose that $S=S e S$ for some idempotent $e$. Then $S$ is locally inverse if and only if it is a locally isomorphic image of a unital regular Rees matrix semigroup over an inverse monoid.

In this case eSe is inverse and $S$ is a locally isomorphic image of a unital regular Rees matrix semigroup over eSe.

COROllary 2.6. Let $S$ be a regular semigroup and let e be an idempotent of $S$. Then $S$ is simple (bisimple) if and only if it is a locally isomorphic image of a unital regular Rees matrix semigroup over a regular simple (bisimple) monoid.

In this case eSe is simple (bisimple) and $S$ is a locally isomorphic image of a unital regular Rees matrix semigroup over $e$ Se.

Corollaries 2.5 and 2.6 depend on a knowledge of Green's relations in regular Rees matrix semigroups. These are easily calculated, as the following lemma shows.

LEMMA 2.7. Let $\Re \mathfrak{R}(S ; I, \Lambda ; P)$ be a regular Rees matrix semigroup over the regular semigroup $S$.

i) $(i, s, \lambda)$ is idempotent if and only if $s=s p_{\lambda i} s$;

ii) let $(i, s, \lambda),(j, t, \mu)$ be idempotents, then

(a) $(i, s, \lambda) \Re(j, t, \mu)$ if and only if $i=j, s \mathscr{R} t$,

(b) $(i, s, \lambda) \mathcal{E}(j, t, \mu)$ if and only if $\lambda=\mu, s \in t$,

(c) $(i, s, \lambda) \omega(j, t, \mu)$ if and only if $i=j, \lambda=\mu$ and $s p_{\lambda_{i}} \omega t p_{\lambda i}$,

(d) $(i, s, \lambda) \mathscr{D}(j, t, \mu)$ if and only if $s \cdot \mathcal{D} t$,

(e) $(i, s, \lambda) \mathcal{g}(j, t, \mu)$ if and only if $s g t$.

Corollary 2.5, coupled with the main theorem of [7], can be used to give a proof of a theorem of Pastijn [11] for principally generated locally inverse semigroups. The proof, in the general case, is much more difficult. 
The homomorphism $\theta$, constructed in Theorem 2.4, is usually far from one to one. The next result specifies exactly when $\theta$ is an isomorphism. We do not need this result so the proof is omitted.

THEOREM 2.8. Let $S=S e S$ be a regular semigroup, where $e^{2}=e$ and let $I, \Lambda$, $P$ be as in Theorem 2.4. Then $\theta: \Re \mathscr{R}(e S e ; I, \Lambda ; P) \rightarrow S$ is an isomorphism if and only if

(i) $u S$ is a maximal principal right ideal of $S$, for each $u \in I$, and dually;

(ii) distinct maximal principal right (left) ideals are disjoint;

(iii) $t \in r_{u}^{\prime} r_{u} S r_{v}^{\prime} r_{v}$ implies $V(t) \cap e S e \subseteq r_{v}^{\prime} r_{v} S r_{u}^{\prime} r_{u}$.

In this case $\Re \mathscr{R}(e S e ; S I, \Lambda ; P)=\left\{(u, s, v): s \in r_{u}^{\prime} r_{u} S r_{v}^{\prime} r_{v}\right\}$.

Condition (iii) in Theorem 2.8 is unsatisfactory since it depends on the choice of elements $r_{u}, r_{u}^{\prime} \in S$. It becomes redundant however in two situations:

(a) $S$ is bisimple; for we may choose $r_{u}^{\prime} r_{u}=e$ in this case;

(b) $S$ is locally inverse; for then $e S e$ is inverse and (iii) is automatic.

Theorem 2.4 leans heavily on the ideas of $D$. Allen [1]. Our contribution consists of defining $\mathfrak{R} \mathscr{R}(S ; I, \Lambda ; P)$ and removing some of Allen's unnecessary hypotheses.

Theorem 2.4 adapts readily to partially ordered semigroups. Suppose that $T$ is a partially ordered regular semigroup and let $I, \Lambda$ be partially ordered sets. Further, suppose that $P$ is an isotone $\Lambda \times I$ matrix over $T$; that is, $P$ is isotone considered as a mapping from $\Lambda \times I$, under the cartesian ordering, into $T$. Then $\Re \mathfrak{R}(T ; I, \Lambda ; P)$ becomes a partially ordered semigroup under

$$
(i, s, \lambda) \leqslant(j, t, \mu) \text { if and only if } i<j, s \leqslant t, \lambda<\mu \text {. }
$$

On the other hand, suppose that $S$ is a partially ordered regular semigroup and that $S=S e S$ for some idempotent $e$. Let $I, \Lambda, P$ be as in the proof of Theorem 2.3 and partially order $I$ and $\Lambda$ by

$$
\begin{array}{ll}
u \leqslant v & \text { for } u, v \in I \text { if and only if } r_{u} \leqslant r_{v}, \\
u \leqslant v & \text { for } u, v \in \Lambda \text { if and only if } r_{u}^{\prime} \leqslant r_{v}^{\prime} .
\end{array}
$$

Then, since $p_{v u}=r_{v}^{\prime} r_{u}, P$ is an isotone matrix over eSe. Further, the map $\theta$ : $(u, s, v) \mapsto r_{u} s r_{v}^{\prime}$ is an isotone mapping of $\mathscr{R} \mathscr{R}(e S e ; I, \Lambda ; P)$ onto $S$. Indeed, restricted to each local submonoid, $\theta$ is an order isomorphism. Hence the analog of Theorem 2.4 also holds in the category of partially ordered regular semigroups.

THEOREM 2.9. Let $S$ be a partially ordered regular semigroup and suppose that $S=S e S$ for some idempotent $e$. Then $S$ is a (partially ordered semigroup) locally isomorphic image of a unital regular Rees matrix semigroup over eSe. 


\section{Naturally ordered regular semigroups with a greatest idempotent}

Let $S$ be a naturally ordered regular semigroup which has a greatest (under the imposed partial order $\leqslant$ ) idempotent $u$. Then, by Corollary $1.6, S=S u S$ so we may apply the local structure theorem to obtain $S$ as a locally isomorphic image of a regular Rees matrix semigroup over an inverse monoid. In order to produce the Rees matrix semigroup, from $S$, we need to choose sets $I, \Lambda$ of idempotents of $S$, and construct the sandwich matrix $P$.

LEMMA 3.1. Let $S$ be a naturally ordered regular semigroup with greatest idempotent $u$. Let $E$ denote the set of idempotents of $S$. Then $E u$ is a set of idempotent representatives of the $R$-classes of $S ; u E$ is a set of idempotent representatives of the $\mathcal{L}$-classes of $S$.

Proof. Let $f \in E$; then $f=f u f$ so that $f \Re f u$ and $f u \in E$. Hence $E u$ contains at least one idempotent from each $R$-class of $S$. Suppose that $f, g \in E u$ and $f \Re g$. Then $u f$ and $u g$ are idempotents in $u E u \subseteq u S u$, which is inverse by Proposition 1.4. Further $u f \Re u g$ since $f \Re g$. Hence $u f=u g$. Thus $f=f u f=f u g$ $=f g$ since $f=f u$. But $f \Re g$ implies $g=f g$ so we must have $f=g$. Hence $E u$ contains exactly one element of each $\Re$-class of $S$; dually $u E$ contains exactly one element of each $\mathcal{L}$-class of $S$.

We let $I=E u, \Lambda=u E$. Then, for $f \in E, f \mathscr{R} f u=r_{f}, f \mathcal{L} u f=r_{f}^{\prime} \in V\left(r_{f}\right)$ and $r_{f} r_{f}^{\prime}=f u f=f, r_{f}^{\prime} r_{f}=u f u \omega u$. Hence, if we let $P$ be the $\Lambda \times I$ matrix with $p_{f, g}=r_{f}^{\prime} r_{g}=u f g u=f g$, then $\theta$ defined by $(e, s, f) \theta=e s f$ is an algebraic local isomorphism of $\Re \mathscr{R}(u S u ; I, \Lambda ; P)$ onto $S$.

Lemma 3.2. For each $(f, g) \in \Lambda \times I, p_{f, g}$ is idempotent.

Proof. Let $B$ denote the subsemigroup generated by the idempotents of $S$. Then $B$ has greatest element $u$ and so, by Lemma 1.7, $u B u$ is a semilattice. Now $f, g \in B$ and so $p_{f, g}=f g=u f g u \in u B u$. Hence $p_{f, g}$ is idempotent.

The sets $I=E u, \Lambda=u E$ being subsets of $S$ inherit a partial order from $S$; each has greatest element $u$. Further the map $P: \Lambda \times I \rightarrow u S u$ is an isotone map and maps $(u, u)$, the greatest element of $\Lambda \times I$ onto the identity of $T=u S u$. Further, when $R=\Re \Re(T ; I, \Lambda ; P)$ is given the cartesian ordering, the mapping $\theta: R \rightarrow S$, defined by $(f, s, g) \theta=f s g$, is a local isomorphism of partially ordered semigroups. Hence we have the converse half of the following theorem. 
THEOREM 3.3. Let $T$ be a naturally ordered inverse monoid and let $\Lambda, I$ be partially ordered sets each with a greatest element (1 say); let $P$ be an isotone map of $\Lambda \times I$ into the idempotents of $T$ such that $p_{1,1}=1$, the identity of $T$. Then, under the cartesian ordering, $\Re \mathscr{R}(T ; I, \Lambda ; P)$ is a naturally ordered regular semigroup with a greatest idempotent.

A partially ordered regular semigroup is naturally ordered with a greatest idempotent if and only if it is a locally isomorphic image of a semigroup constructed in this way.

Proof. It is straightforward to show that $\Re \mathscr{R}(T ; I, \Lambda ; P)$ is a naturally ordered regular semigroup with greatest idempotent $(1,1,1)$. Suppose that $S$ is any isotone image of $\mathscr{R} \mathscr{R}(T ; I, \Lambda ; P)$, say by a homomorphism $\theta$. Suppose that $\bar{e}, \bar{f}$, are idempotents in $S$ such that $\bar{e} \omega \bar{f}$. Then, by Lallement's Lemma ([6], p. 52), twice, there are idempotents $e, f \in \Re \Re(T ; I, \Lambda ; P)$ such that $e \theta=\bar{e}, f \theta=\bar{f}$ and ewf. It follows that $e \leqslant f$, since $R \mathscr{R}(T ; I, \Lambda ; P)$ is naturally ordered, and thus $\bar{e}=e \theta \leqslant f \theta=\bar{f}$. Hence $S$ is naturally ordered. It clearly has a greatest idempotent.

COROllaRY 3.4 (to the proof). If $S$ is a naturally ordered regular semigroup, so is any isotone homomorphic image of $S$.

COROLlaRy 3.5. Let $T$ be a semilattice with greatest element 1 and let $\Lambda, I$ be partially ordered sets each with greatest element 1 , say; let $P$ be an isotone map of $\Lambda \times I$ into $T$ such that $p_{1,1}=1$. Then, under the cartesian ordering, $\Re \Re(T ; I, \Lambda ; P)$ is a naturally ordered regular semigroup with a greatest element.

A partially ordered regular semigroup is naturally ordered with a greatest element if and only if it is a locally isomorphic image of a semigroup constructed as above.

Proof. It is straightforward to show that $\mathscr{R} \mathscr{R}(T ; I, \Lambda ; P)$ is naturally ordered and, clearly, it has greatest element $(1,1,1)$. Further any isotone image of a partially ordered semigroup with a greatest element also has a greatest element. And, by Corollary 3.4, any isotone image of a naturally ordered regular semigroup is naturally ordered.

On the other hand, if $S$ is naturally ordered with greatest elements $u$ then, by Theorem 3.3 , it is a locally isomorphic image of $\mathscr{R} \mathscr{R}(u S u ; I, \Lambda ; P)$, where $I=E u, \Lambda=u E$ and $E$ is the set of idempotents of $S$. Further, by Lemma 1.7, $u S u$ is a semilattice.

COROLlary 3.6. A simple regular semigroup gan be naturally ordered with a greatest element if and only if it is a rectangular band. 
Proof. Suppose $S$ is a naturally ordered simple regular semigroup with greatest element $u$. Then $S$ is a locally isomorphic image of a regular Rees matrix semigroup over $u S u$, where $u S u$ is a semilattice. But $S$ simple implies $u S u$ simple and thus, since it is a semilattice, $u S u$ is trivial. But then any Rees matrix semigroup over $u S u$ is a rectangular band. Hence, so is $S$.

Conversely it is clear that any rectangular band can be partially ordered in the required way.

An analogous result to Corollary 3.5 holds for Dubreil-Jacotin regular semigroups which are naturally ordered. The proof is, however, somewhat more intricate so we state this result as a separate theorem.

THEOREM 3.7. Let $T$ be a Dubreil-Jacotin inverse monoid, with greatest idempotent the identity 1 . Let $I, \Lambda$ be partially ordered sets each with a greatest element, 1 say, and let $P$ be an isotone map of $\Lambda \times I$ into the semilattice of idempotents of $T$ such that $p_{1,1}=1$. Then $\Re \mathscr{R}(T ; I, \Lambda ; P)$ is a naturally ordered Dubreil-Jacotin regular semigroup, under the cartesian ordering.

A partially ordered regular semigroup is a naturally ordered Dubreil-Jacotin semigroup if and only if it is a locally isomorphic image of a semigroup constructed as above.

Proof. Let $T, I, \Lambda, P$ be as above and let $\theta$ denote the natural homomorphism of $T$ onto its maximum group homomorphic image $G$. Then $G$ is partially ordered by $a \theta<b \theta$ if and only if $e a<e b$ for some idempotent $e$ and $\theta$ is an isotone homomorphism of $T$ onto $G$ such that $a \theta<1 \theta$ if and only if $a<1$.

Define a mapping $\varphi: \Re \Re(T ; I, \Lambda ; P) \rightarrow G$ by setting $(i, s, \lambda) \varphi=s \theta$. Then, since each $p_{\lambda i}$ is idempotent, it is immediate that $\varphi$ is an isotone homomorphism of $\Re \mathscr{R}(T ; I, \Lambda ; P)$ onto the partially ordered group $G$. Further, $(i, s, \lambda) \varphi<1 \theta$, the identity of $G$, implies $s \theta \leqslant 1 \theta$ and so, since $T$ is a Dubreil-Jacotin semigroup, $s<1$. Hence $(i, s, \lambda) \leqslant(1,1,1)$ so that $\Re \mathscr{N}(T ; I, \Lambda ; P)$ is a DubreilJacotin semigroup; we have already seen that it is naturally ordered.

On the other hand, suppose that $S$ is a naturally ordered Dubreil-Jacotin regular semigroup and let $u$ be the greatest idempotent of $S$. Then $u S u$ is a Dubreil-Jacotin inverse monoid and $S$ is a locally isomorphic image of $\Re \Re(u S u ; I, \Lambda ; P)$, for some $I, \Lambda, P$.

To complete the proof of the theorem, we show that any locally isomorphic image of a naturally ordered Dubreil-Jacotin regular semigroup is itself a Dubreil-Jacotin semigroup. To this end, let $T$ be a naturally ordered DubreilJacotin regular semigroup and let $\theta$ be a local isomorphism (of partially ordered semigroups) of $T$ onto a partially ordered semigroup $S$. Denote by $\varphi$ the, unique, 
isotone homomorphism of $T$ onto a group $G$ such that $\{x \in T: x \varphi<1$, the identity of $G$ \} has a greatest element $v$ and let $u=v \theta$; then $u$ is the greatest idempotent of $S$, since $\theta$ is isotone.

Suppose that $a \theta=b \theta$ then, for each $b^{\prime} \in V(b), a b^{\prime} \theta=\left(b b^{\prime}\right) \theta$ so that, by Lemma 2.3, $a b^{\prime}$ is idempotent, because $\theta$ is a local isomorphism. Hence $\left(a b^{\prime}\right) \varphi=$ 1 ; that is $a \varphi=b \varphi$. It follows therefore that there is a unique homomorphism $\psi$ : $S \rightarrow G$ such that $\varphi=\theta \psi$.

Suppose that $a \theta \leqslant b \theta$. Then $\left(b b^{\prime} a b^{\prime}\right) \theta \leqslant b b^{\prime} \theta$, for $b^{\prime} \in V(b)$ where $b b^{\prime} a b^{\prime}$ and $b b^{\prime}$ both belong to $b b^{\prime} T b b^{\prime}$. Hence, since $\theta$ is a local isomorphism, $b b^{\prime} a b^{\prime}<b b^{\prime}$ and thus $\left(b b^{\prime} a b^{\prime}\right) \varphi \leqslant\left(b b^{\prime}\right) \varphi$. This implies $\left(b b^{\prime} a b^{\prime} b\right) \theta \psi \leqslant b \theta \psi$ and so, since $G$ is a group, $a A \psi \leqslant b \theta \psi$. Hence $\psi$ is isotone and further

$$
\begin{aligned}
a \theta \psi \leqslant 1 & \text { if and only if } a \varphi<1, \\
& \text { if and only if } a<v, \\
& \text { if and only if } a \theta<u
\end{aligned}
$$

since $\psi$ is isotone and $u$ is idempotent. Thus $S$ is a Dubreil-Jacotin regular semigroup and, since $T$ is naturally ordered, so is $S$.

\section{References}

[1] D. Allen, Jr., 'A generalization of the Rees theorem to a class of regular semigroups', Semigroup Forum 2 (1971), 321-331.

[2] T. S. Blyth, 'Dubreil-Jacotin inverse semigroups', Proc. Roy. Soc. Edinburgh Sect. A 71 (1974), 345-360.

[3] _ 'The structure of certain ordered regular semigroups', Proc. Roy. Soc. Edinburgh Sect. A 75 (1976), 235-257.

[4] _ 'On a class of Dubreil-Jacotin semigroups and a construction of Yamada', Proc. Roy. Soc. Edinburgh Sect. A 77 (1977), 145-150.

[5] , 'Perfect Dubreil-Jacotin semigroups', Proc. Roy. Soc. Edinburgh Sect. A 78 (1977), $101-104$.

[6] J. M. Howie, An introduction to semigroup theory (Academic Press, London, 1976).

[7] D. B. McAlister, 'Groups, semilattices and inverse semigrups, II', Trans. Amer. Math. Soc. 196 (1974), 351-370.

[8] D. B. McAlister and T. S. Blyth, 'Split orthodox semigroups', J. Algebra 51 (1978), 491-525.

[9] K. S. S. Nambooripad, 'Structure of regular semigroups I', Mem. Amer. Math. Soc. 22, Number 224 (1979).

[10] _ The natural partial order on a regular semigroup', to appear.

[11] F. J. Pastijn, 'Structure theorems for pseudo inverse semigroups', Proceedings of a Sympasium on Regular Semigroups, Northern Illinois University, April 1979.

Department of Mathematical Sciences

Northern Illinois University

DeKalb, Illinois 60115

U.S.A. 